\title{
Nuclear magnetic relaxation rate dispersion in supercooled heavy water under high pressure ${ }^{\text {a) }}$
}

\author{
E. W. Lang and H.-D. Lüdemann \\ Institut für Biophysik und Physikalische Biochemie, Universität Regensburg, D 8400 Regensburg 2, \\ Federal Republic of Germany \\ L. Piculell \\ Division of Physical Chemistry 1, Chemical Center, P. O. Box 740, S-22007 Lund 7, Sweden
}

(Received 10 February 1984; accepted 27 June 1984)

\begin{abstract}
Spin-lattice $\left(T_{1}\right)$ and spin-spin $\left(T_{2}\right)$ relaxation times of the deuterons in supercooled $\mathrm{D}_{2} \mathrm{O}$ at 225 $\mathrm{MPa}$, measured at two frequencies: 55.54 and $39.14 \mathrm{MHz}$ down to $188 \mathrm{~K}$ are reported. The results show that $T_{1}$ and $T_{2}$ become frequency dependent in supercooled liquid water under high hydrostatic pressure at temperatures below $\sim 220 \mathrm{~K}$. Theoretical expressions for the relaxation rates are deduced under the assumption that the orientational fluctuations of the water molecules are composed of fast librational oscillations and slower diffusional motions. The effect of the librations is to reduce the size of the deuterium quadrupole coupling constant. The diffusional motions are nearly isotropic and dominate the $T$ dependence of the relaxation times. The autocorrelation function of the slow orientational fluctuations was assumed to be exponential at long times with a single time constant, the orientational correlation time $\tau_{2}$. The $T$ dependence of the latter is well described by the VTF equation. The parameters obtained by least squares fitting the experimental spin-lattice relaxation times to an isotropic motional model correctly predict the temperature and frequency dependence of the spin-spin relaxation times.
\end{abstract}

\section{INTRODUCTION}

The unusual physical properties of supercooled water have been the subject of many experimental and theoretical studies in the last years. ${ }^{1}$ Since most of these anomalies tend to be more accentuated in close proximity of the homogeneous nucleation temperature $T_{H}$, it is important to extend any measurements as close as possible to this temperature. Since all studies in the extremely supercooled region have been performed on water emulsions, the dynamics of liquid water have hitherto only been studied by NMR relaxation rate measurements. ${ }^{2-5}$ When applied to the ${ }^{17} \mathrm{O}$ and ${ }^{2} \mathrm{H}$ nuclei, the relaxation rates monitor single molecule reorientation rather directly. The information is contained in the spectral density function of these motions. As the NMR technique is a resonance experiment, the spectral density function is probed at the resonance frequency of the experiment only. At ambient temperatures the reorientational correlation function of the water molecule decays rapidly on the NMR time scale thus rendering the spectral density function frequency independent in the accessible frequency range. It has been shown recently ${ }^{2,3,6}$ that at low temperatures $(T \leqslant 220 \mathrm{~K})$ the reorientation of water molecules in the supercooled liquid is slowed down sufficiently for the relaxation rates to become frequency dependent. To achieve such low temperatures, one has to undercool water under an external pressure where $T_{H}$ is at its minimum, which for $\mathrm{D}_{2} \mathrm{O}$ occurs at $225 \mathrm{MPa}^{1}$

In the present paper we report spin-lattice $\left(T_{1}\right)$ and spin-spin $\left(T_{2}\right)$ relaxation times of the deuterons in supercooled $\mathrm{D}_{2} \mathrm{O}$ at $225 \mathrm{MPa}$, measured at two frequencies $(55.54$ and $39.14 \mathrm{MHz}$ ) down to $188 \mathrm{~K}$. Including previous data at

\footnotetext{
a) The ordering of authors' names in this cooperative investigation is not significant.
}

15.35 $\mathrm{MHz}^{3}$ one can now probe the spectral density function at four frequencies. The combined results thus provide a more stringent test of any model of liquid water which has dynamical implications than the results available before.

\section{EXPERIMENTAL}

Deuteron magnetic relaxation times were measured at $55.54 \mathrm{MHz}$ on a Nicolet NIC-360 FT spectrometer and at $39.14 \mathrm{MHz}$ on a home-built FT spectrometer equipped with a $6 \mathrm{~T}$ magnet. Spin-lattice relaxation times $\left(T_{1}\right)$ were measured by the alternating phase inversion recovery method (APIRFT). ${ }^{7}$ Spin-spin relaxation times were obtained from the linewidth at half-amplitude $(\Delta v)$ of absorption spectra according to $T_{2}^{-1}=\pi \cdot \Delta v$. The linewidths were corrected for contributions from magnetic field inhomogeneity evaluated at the lowest temperatures where $T_{1}=T_{2}(39.14 \mathrm{MHz}$ : $12 \mathrm{~Hz}$ at $222 \mathrm{~K} ; 55.54 \mathrm{MHz}: 19 \mathrm{~Hz}$ at $230 \mathrm{~K}$ ). From reproducibility considerations we estimate an uncertainty of $\pm 5 \%\left(T_{1}\right)$ and $\pm 6 \%(\Delta v)$ resulting in an uncertainty of $\pm 11 \%$ in $T_{2}$.

The undercooling of water is considerably facilitated if water is emulgated in a cycloalcane/surfactant mixture. ${ }^{8}$ The emulsions, which were prepared as described earlier, ${ }^{3}$ were contained in a high pressure glass capillary with i. d. 1.2 $\mathrm{mm}$ and o. d. $7 \mathrm{~mm}$. The high pressure NMR cell used is a slightly modified version of the one described previously ${ }^{9}$ (see Fig. 1). The standard spinner housing of the spectrometers was replaced by a Teflon cell holder (see Fig. 1) which fitted tightly into the bore of the superconducting magnets. The Teflon holder was screwed onto an aluminum tube, 0.8 $\mathrm{m}$ long, with five spacers fixed at equal distance in order to center the high pressure cell exactly in the bore. The sample temperature was controlled by the passage of thermostated nitrogen through the probe and was measured by a copper- 


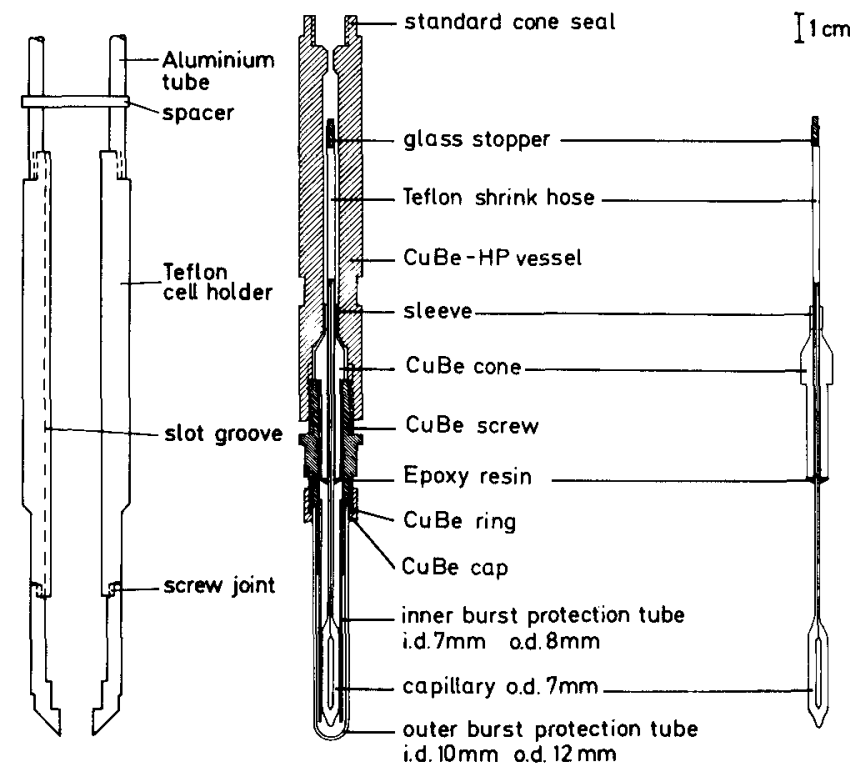

FIG. 1. High pressure NMR cell with glass capillary and Teflon cell holder.

Constantan thermocouple (o. d. $0.9 \mathrm{~mm}$ ) before every $T_{1}$ measurement. The thermocouple was guided by a stiff Teflon shrinkhose inside the aluminum tube. This shrinkhose was fixed in a slot of the Teflon cell holder and glued onto the $\mathrm{Cu}-\mathrm{Be}$ cell with a sticker. The thermocouple could then be pushed through a groove in the $\mathrm{Cu}-\mathrm{Be}$ screw into the space between the inner and the outer protection glass tubes and positioned at the height of the receiver coil. During the experiment the thermocouple was pulled up by about $0.1 \mathrm{~m}$. The temperatures measured are considered reliable to \pm 0.5 K.

The applied pressure was measured by a precision Bourdon gauge (Heise, Newton, CT) to $\pm 0.5 \mathrm{MPa}$ and was generated with standard 1/8 in. equipment (HIP, Erie, PA).

\section{RESULTS}

Table I contains the spin-lattice $\left(T_{1}\right)$ and spin-spin $\left(T_{2}\right)$ relaxation times measured at 39.14 and $55.54 \mathrm{MHz}$. The spin-lattice relaxation times are shown in an Arrhenius diagram in Fig. 2. Included are the data from Lang and Lüdemann $^{3}$ at $15.35 \mathrm{MHz}$. At temperatures $T \gtrsim 220 \mathrm{~K} T_{1}$ is independent of the observing frequency $\omega_{0}$ and is in good agreement with results obtained previously. ${ }^{3}$ At each observing frequency $\omega_{0}$ the longitudinal relaxation times $T_{1}$ exhibit a minimum which occurs nearer to the homogeneous nucleation temperature $T_{H}$ the lower the frequency $\omega_{0}$. The spin-spin relaxation times $T_{2}$ obtained in the dispersion region $(T \leqslant 220 \mathrm{~K})$ are contained in the insert of Fig. 2. Contrary to the behavior of $T_{1}$, the transverse relaxation time $T_{2}$ continues to decrease with falling temperature.

\section{THEORY}

The deuterium spin relaxation is dominated by the interaction of the nuclear quadrupole moment $e Q$ with the electric field gradient (efg) at the nucleus. ${ }^{10}$ There is no evidence for significant contributions from either chemical exchange of the deuterons or from dipolar mechanisms. The

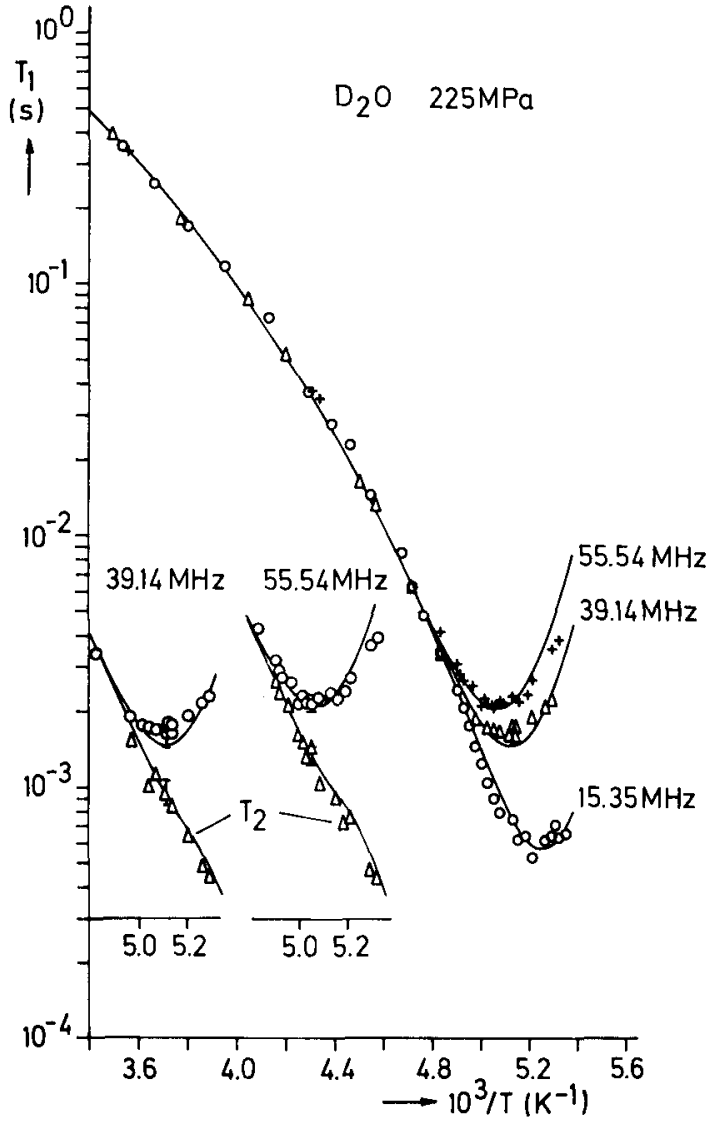

FIG. 2. Experimental spin-lattice relaxation times $\left(T_{1}\right)$ obtained at 55.54 $(+)$ and $39.14 \mathrm{MHz}(\triangle)$ together with previous data measured at 15.35 $\mathrm{MHz}(\mathrm{O})$ (see Ref. 3). The solid lines represent best fit curves to Eq. (6) with $\mathrm{DQC}_{\mathrm{eff}}=201.1 \mathrm{kHz}, \tau_{20}=4.98 \times 10^{-14} \mathrm{~s}, B=687.6 \mathrm{~K}$, and $T_{0}=132 \mathrm{~K}$. Insert: Experimental spin-lattice $(O)$ and spin-spin $(\triangle)$ relaxation times obtained at 55.54 and $39.14 \mathrm{MHz}$. Solid lines represent the $T$ dependence of the relaxation times as given by Eq. (6) for $T_{1}$ and Eq. (7) for $T_{2}$ together with Eq. (14) for the correlation times $\tau_{2}$.

efg at a given nucleus is the sum of electronic and nuclear contributions. ${ }^{11-13}$ The electronic contribution to the field gradient at the deuteron arises almost entirely from centers other than the deuteron itself, because the $2 s$ orbital at the deuteron makes no contribution. In liquid water the efg is determined predominantly by the O-D bond length. ${ }^{14}$ It is thus a reasonable approximation, at least for weak, asymmetric $\mathrm{H}$ bonds, to regard the field gradient as being generated exclusively by intramolecular charges.

The relevant interaction Hamiltonian is given in a laboratory-fixed frame $(L)$ as ${ }^{15}$

$$
H_{Q}(t)=\frac{e Q}{2 I(2 I-1) h} \sum_{m=-2}^{2}(-1)^{m} T_{2 m}^{L} R_{2-m}^{L}(t) .
$$

$I$ is the nuclear spin quantum number and $e Q$ is the nuclear quadrupole moment. The $T_{2 m}$ are second rank irreducible spin tensor operators and the $R_{2 m}$ are components of the second rank irreducible field gradient tensor. The latter are most conveniently expressed in a molecule fixed principal axis system $(I)$. The transformation to the laboratory-fixed frame can be effected with the aid of the second rank Wigner rotation matrix. ${ }^{16}$

To generate the appropriate transformation one has to consider the rotational dynamics of the water molecules in 
TABLE I. Experimental spin-lattice $\left(T_{1}\right)$ and spin-spin $\left(T_{2}\right)$ relaxation times measured at $p=225 \mathrm{MPa}$ and at the observing frequencies: 55.54 and 39.14 MHz.

\begin{tabular}{|c|c|c|c|c|c|}
\hline$T \pm 0.5(\mathbf{K})$ & $\omega_{0} / 2 \pi(\mathrm{MHz})$ & $T_{1} \pm 5 \%(\mathrm{~ms})$ & $T_{2} \pm 9 \%(\mathrm{~ms})$ & $\tau_{2} \pm 20 \%(\mathrm{~ns})^{n}$ & $\mathrm{DQC}_{\text {eff }} \pm 20 \%(\mathrm{kHz})^{\mathrm{b}}$ \\
\hline 286 & 39.14 & 390 & & & \\
\hline 281 & 55.54 & 336 & & & \\
\hline 265 & 39.14 & 179 & & & \\
\hline 247 & 39.14 & 85 & & & \\
\hline 238 & 39.14 & 52 & & & \\
\hline 232 & 55.54 & 38 & & & \\
\hline 232 & 55.54 & 36 & & & \\
\hline 230.5 & 55.54 & 35 & & & \\
\hline 222 & 39.14 & 16 & & & \\
\hline $\begin{array}{l}219 \\
212\end{array}$ & $\begin{array}{l}39.14 \\
39.14\end{array}$ & $\begin{array}{l}13 \\
6.25\end{array}$ & & & \\
\hline $\begin{array}{l}212 \\
207\end{array}$ & 39.14 & $\begin{array}{l}0.30 \\
3.31\end{array}$ & & & \\
\hline 207 & 55.54 & 4.14 & & & \\
\hline 204 & 55.54 & 3.08 & 3.07 & & \\
\hline 203.5 & 55.54 & 2.81 & 2.55 & 0.65 & 209 \\
\hline 203 & 55.54 & 2.66 & 2.34 & 0.76 & 203 \\
\hline 201.5 & 55.54 & 2.54 & 2.05 & 1.03 & 192 \\
\hline 201 & 39.14 & 1.87 & 1.48 & 1.54 & 185 \\
\hline 200 & 55.54 & 2.10 & 1.56 & 1.26 & 203 \\
\hline 199.5 & 55.54 & 2.26 & & & \\
\hline 199 & 55.54 & 2.12 & 1.49 & 1.42 & 199 \\
\hline 199 & 39.14 & 1.73 & & & \\
\hline 198.5 & 55.54 & 2.13 & 1.31 & 1.81 & 197 \\
\hline 198.5 & 55.54 & 2.22 & & & \\
\hline 198.5 & 55.54 & 2.00 & & & \\
\hline 198 & 55.54 & 2.14 & 1.37 & 1.69 & 197 \\
\hline 198 & 55.54 & 2.00 & 1.31 & 1.62 & 204 \\
\hline 198 & 39.14 & 1.59 & 1.07 & 2.19 & 192 \\
\hline 198 & 39.14 & 1.76 & 1.03 & 2.77 & 182 \\
\hline 198 & 39.14 & 1.72 & 0.869 & 3.42 & 188 \\
\hline 198 & 39.14 & 1.72 & 0.964 & 2.96 & 185 \\
\hline 197.7 & 55.54 & 2.12 & 1.29 & 1.84 & 198 \\
\hline 197.7 & 55.54 & 2.00 & & & \\
\hline 197.7 & 55.54 & 2.22 & 1.51 & 1.52 & 194 \\
\hline 197 & 55.54 & 2.21 & & & \\
\hline 197 & 39.14 & 1.63 & 1.08 & 2.22 & 190 \\
\hline 197 & 39.14 & 1.64 & & & \\
\hline 197 & 39.14 & 1.67 & & & \\
\hline 197 & 39.14 & 1.68 & & & \\
\hline \multirow[t]{2}{*}{196.4} & 55.54 & 2.07 & 0.858 & 3.08 & 212 \\
\hline & 55.54 & 2.25 & 1.15 & 2.38 & 195 \\
\hline 195.5 & 39.14 & 1.60 & 0.925 & 2.80 & 191 \\
\hline 195 & 55.54 & 2.31 & & & \\
\hline 195 & 39.14 & 1.75 & & & \\
\hline 194.6 & 39.14 & 1.72 & & & \\
\hline 194.4 & 39.14 & 1.60 & 0.82 & 3.38 & 194 \\
\hline 194 & 55.54 & 2.19 & 0.858 & 3.28 & 209 \\
\hline 194 & 55.54 & 2.30 & 0.925 & 3.18 & 203 \\
\hline 194 & 55.54 & 2.04 & 0.845 & 3.08 & 214 \\
\hline 192.7 & 55.54 & 2.52 & 0.708 & 4.54 & 214 \\
\hline 192.7 & 55.54 & 2.32 & & & \\
\hline 192.7 & 55.54 & 2.19 & & & \\
\hline 192 & 55.54 & 2.67 & 0.725 & 4.68 & 210 \\
\hline 192 & 39.14 & 1.97 & 0.635 & 5.68 & 195 \\
\hline 192 & 39.14 & 1.78 & 0.630 & 5.21 & 200 \\
\hline 190 & 39.14 & 2.11 & 0.460 & 7.90 & 210 \\
\hline 189 & 39.14 & 2.25 & 0.430 & 8.79 & 212 \\
\hline 189 & 55.54 & 3.58 & 0.450 & 8.23 & 225 \\
\hline 188 & 55.54 & 3.85 & 0.423 & 8.95 & 225 \\
\hline
\end{tabular}

Orientational correlation times $\tau_{2}$ obtained from the ratio $T_{1} / T_{2}$ [Eqs. (6) and (7)].

${ }^{b}$ Effective deuterium quadrupole coupling constant calculated with Eq. (6) and $\tau_{2}$ as obtained from the ratio $T_{1} / T_{2}$.

the liquid state. In the random network model ${ }^{17}$ liquid water at low temperatures is described as a continuous random network of hydrogen-bonded molecules with their nearest neighbor arrangement corresponding on the average to a te- trahedral coordination. Molecular motions are divided into fast oscillatory motions and slower diffusional processes. The short time reorientational motions of the water molecule consist of fast librations about an equilibrium orienta- 
tion in the quasistatic random network. On a longer time scale this equilibrium orientation will change due to slower rotational diffusive motions thereby leading to a permanent rearrangement of the network topology. With this picture of molecular motion in liquid water occurring on two different time scales, it is convenient to perform the coordinate transformations $I \rightarrow L$ via an additional coordinate system, and to take into consideration the time dependence of each of the transformations separately. ${ }^{18}$ Thus the following coordinate systems are introduced: The principal axis system $(I)$ of the field gradient tensor fixed in the molecule with its $z$ axis in the direction of the OD bond, an axis system whose $z$ axis describes the equilibrium orientation of the OD bond $(F)$ in the quasistatic network and the laboratory-fixed axis system $(L)$. The orientation of each coordinate system will be specified by three Euler angles according to Rose. ${ }^{16}$ The transformation $I \rightarrow F$ is time dependent due to molecular librations. The components of the electric field gradient tensor are considered to be time independent in the principal axis system $I$. The transformation $F \rightarrow L$ is time dependent due to rotational diffusive motions of the molecules. With these transformations the $R_{2 m}^{L}$ are given by

$$
R_{2 m}^{L}=\sum_{m^{\prime}} D_{m^{\prime} m}^{2}\left(\Omega_{F L}, t\right) \sum_{m^{\prime}} D_{m^{\prime \prime}, m^{\prime}}^{2}\left(\Omega_{I F} t\right) \rho_{m^{*}},
$$

where $\rho_{m}$ denotes the spherical components of the electric field gradient tensor in the principal axis system $I .{ }^{15}$ From the $R_{20}^{L}$ one obtains their autocorrelation functions $f_{20}$ in an isotropic liquid as

$$
\begin{aligned}
f_{20}(\tau)= & \left\langle R_{20}^{*}(\tau) R_{20}(0)\right\rangle \\
= & \left\langle\sum_{m} \sum_{m^{\prime}} D_{m 0}^{(2) *}\left(\Omega_{F L}, \tau\right) D_{m^{\prime} m}^{(2) *}\left(\Omega_{I F}, \tau\right) \rho_{m^{\prime}}\right. \\
& \left.\times \sum_{n} \sum_{n^{\prime}} D_{n 0}^{(2)}\left(\Omega_{F L}, 0\right) D_{n^{\prime} n}^{(2)}\left(\Omega_{I F}, 0\right) \rho_{n^{\prime}}\right\rangle,
\end{aligned}
$$

where \langle\rangle denotes either a time average or an ensemble average. Since the fast librational motions occur on a much shorter time scale than the slower diffusive tumbling there is no cross correlation. ${ }^{18,54,55}$ Hence these motions are statistically independent and one can factorize the correlation function. ${ }^{52,53}$ This is rigorously true if the slow diffusive reorientation is isotropic and constitutes a Markov process with a single time constant $\tau_{2}$. As the librational correlation function decays much faster than the rotational correlation function $^{21}$ the librational motions are uncorrelated for times longer than the rotational correlation time $\tau_{2}$. With the general behavior of time correlation functions in the limit $t \rightarrow \infty^{20} \lim _{t \rightarrow \infty} G_{A B}(t)=\langle A\rangle\langle B\rangle$ we finally obtain

$$
\begin{aligned}
f_{2}(\tau)= & \frac{1}{5} \exp \left(-\frac{\tau}{\tau_{2}}\right) \cdot \frac{3}{2}\left(e q_{z z}^{I}\right)^{2} \sum_{m} \mid\left\langle D_{0 m}^{(2)}\left(\Omega_{I F}\right)\right\rangle \\
& +\left.\frac{\eta}{\sqrt{6}}\left[\left\langle D_{2 m}^{(2)}\left(\Omega_{I F}\right)\right\rangle+\left\langle D_{-2 m}^{(2)}\left(\Omega_{I F}\right)\right\rangle\right]\right|^{2} \\
= & \frac{3}{10} e^{2} q_{\mathrm{eff}}^{2} \exp \left(-\tau / \tau_{2}\right) .
\end{aligned}
$$

The Fourier-Laplace transform of the autocorrelation function $f_{2}(\tau)$ is the spectral density $g_{2}\left(\omega_{0}\right)$, given by ${ }^{15}$

$$
g_{2}\left(m \omega_{0}\right)=\int_{0}^{\infty} f_{2}(\tau) \exp \left(i m \omega_{0} \tau\right) d \tau \equiv f_{2}(0) \cdot \tilde{g}_{2}\left(m \omega_{0}\right),
$$

where the last step defines the reduced spectral density $\tilde{g}_{2}\left(\omega_{0}\right)$. The spin lattice $\left(1 / T_{1}\right)$ and spin-spin $\left(1 / T_{2}\right)$ relaxation rates of the deuterium nucleus can be expressed as

$$
\begin{aligned}
& \frac{1}{T_{1}}=\frac{3 \pi^{2}}{20}\left(\frac{e^{2} q_{\mathrm{eff}} Q}{h}\right)^{2} \cdot F_{2}\left(\omega_{0}, \tau_{2}\right), \\
& \frac{1}{T_{2}}=\frac{3 \pi^{2}}{20}\left(\frac{e^{2} q_{\mathrm{eff}} Q}{h}\right)^{2} \cdot F_{2}^{\prime}\left(\omega_{0}, \tau_{2}\right),
\end{aligned}
$$

where

$$
\begin{aligned}
& F_{2}\left(\omega_{0}, \tau_{2}\right)=2 \tilde{g}_{2}\left(\omega_{0}\right)+8 \tilde{g}_{2}\left(2 \omega_{0}\right), \\
& F_{2}^{\prime}\left(\omega_{0}, \tau_{2}\right)=3 \tilde{g}_{2}(0)+5 \tilde{g}_{2}\left(\omega_{0}\right)+2 \tilde{g}_{2}\left(2 \omega_{0}\right), \\
& \tilde{g}_{2}\left(m \omega_{0}\right)=\frac{\tau_{2}}{1+\left(m \omega_{0} \tau_{2}\right)^{2}},
\end{aligned}
$$

and with Eq. (4) inserted in Eq. (5). Both expressions [(6) and (7)] reduce in the extreme narrowing limit $\left(\omega_{0}^{2} \tau_{2}^{2}<1\right)$ to

$$
\frac{1}{T_{1}}=\frac{1}{T_{2}}=\frac{3 \pi^{2}}{2}\left(\frac{e^{2} q_{\mathrm{eff}} Q}{h}\right)^{2} \cdot \tau_{2}
$$

rendering $T_{1}=T_{2}$.

The sole effect of the fast oscillations is thus to reduce the deuterium quadrupole coupling constant $D Q C$ from its instantaneous value

$$
\mathrm{DQC} \equiv \frac{e^{2} q_{z z}^{I} Q}{h}
$$

to a, librationally averaged, value

$$
\mathrm{DQC}_{\mathrm{eff}}=\frac{e^{2} q_{\mathrm{eff}} Q}{h} \equiv \frac{e^{2} q_{z z}^{I} Q}{h} \cdot \Gamma .
$$

The motional averaging factor $\Gamma$ is given by ${ }^{54,55}$

$$
\begin{aligned}
\Gamma^{2}= & \sum_{m} \mid\left\langle D_{o m}^{(2)}\left(\Omega_{I F}\right)\right\rangle+\frac{\eta}{\sqrt{6}}\left[\left\langle D_{-2 m}^{(2)}\left(\Omega_{I F}\right)\right\rangle\right. \\
& \left.+\left\langle D_{2 m}^{(2)}\left(\Omega_{I F}\right)\right\rangle\right]\left.\right|^{2}
\end{aligned}
$$

For the deuteron in water, the efg asymmetry parameter $\eta \equiv\left(q_{x x}^{I}-q_{y y}^{I}\right) / q_{z z}^{I}$ is small $(\eta \simeq 0.1$ in both the solid and the vapor phase of $\mathrm{D}_{2} \mathrm{O}^{19}$ ) so only the first term in the summand of Eq. (11) needs to be retained.

\section{DISCUSSION}

\section{Orientational correlation times}

Applying Eqs. (6) and (7) to the experimental results, one deduces from Eq. (6) that the $T_{1}(T)$ curve will run through a minimum - as observed experimentally-whenever $\tau_{2}(T)=0.6158 \cdot \omega_{0}^{-1}$ regardless of the value of $\mathrm{DQC}_{\mathrm{eff}}$ at that temperature. Thus one can calculate precise correlation times at the temperatures $T_{\min }\left(\omega_{0}\right)$ of the corresponding $T_{1}$ minima (see Table II). At $T_{\min }\left(\omega_{0}\right)$ one can also calculate, without further assumptions, the ratio $T_{1} / T_{2}$. Equations (6) and (7) yield $T_{1} / T_{2}=1.60$ and the experimental ratios, given in Table II, are seen to be in excellent agreement with the theoretical prediction. Addressing the question whether the experimental data indicate any contribution from the fast librations to $g\left(\omega_{0}\right)$ beyond that already incorporated in Eqs. 
TABLE II. Spin-lattice $\left(T_{1}\right)$ and spin-spin $\left(T_{2}\right)$ relaxation times obtained at the temperatures $T=T_{\min }\left(\omega_{0}\right)$.

\begin{tabular}{llllllll}
\hline \hline$\omega_{0} / 2 \pi(\mathrm{MHz})$ & $T_{\min }(\mathrm{K})$ & $T_{1}\left(T_{\min }\right)(\mathrm{ms})$ & $T_{2}\left(T_{\min }\right)(\mathrm{ms})$ & $T_{1} / T_{2}$ & $\tau_{2}(\mathrm{~ns})^{\mathrm{a}}$ & $\tau_{2}(\mathrm{~ns})^{\mathrm{b}}$ & $\mathrm{DQC}$ es $(\mathrm{kHz})^{\mathrm{c}}$ \\
\hline 55.54 & $198 \pm 2$ & $2.07 \pm 0.10$ & $1.34 \pm 0.14$ & $1.6 \pm 0.2$ & $1.7 \pm 0.2$ & 1.765 & $200 \pm 6$ \\
39.14 & $195 \pm 2$ & $1.60 \pm 0.08$ & $0.93 \pm 0.09$ & $1.7 \pm 0.2$ & $2.8 \pm 0.3$ & 2.504 & $191 \pm 6$ \\
$15.35^{\mathrm{d}}$ & $191 \pm 2$ & $0.50 \pm 0.06$ & & & & 6.385 & $214 \pm 12$ \\
\hline \hline
\end{tabular}

"Deduced from the ratio $T_{1} / T_{2}$ according to Eqs. (6) and (7).

${ }^{b}$ Calculated from the condition $\omega_{0} \cdot \tau_{2}=0.6158$.

(6) and (7), the numerical agreement of theory and experiment leads one to suspect that any additional contribution would be negligibly small at these (and higher) temperatures. As a consequence, the $T$ dependence of $T_{1}$ and $T_{2}$ is dominated by the orientational correlation time $\tau_{2}$. Additional correlation times $\tau_{2}$ can be calculated in the dispersion region from the ratio $T_{1} / T_{2}$ at any given temperature and pressure without knowledge of $\mathrm{DQC}$ eff. The correlation times $\tau_{2}$ obtained in this manner are given in Table $I$ and their $T$ dependence is shown in Fig. 3. The values extracted from $T_{1} / T_{2}$ are in excellent agreement with those calculated using the condition $\tau_{2}\left[T_{\min }\left(\omega_{0}\right)\right]=0.6158 \cdot \omega_{0}^{-1}$ (see Table II). A comparison with correlation times at ambient temperatures, where $\tau_{2} \simeq 10^{-12} \mathrm{~s}^{3}$ shows that the reorientational mobility of water is slowed down by 3-4 orders of magnitude at these low temperatures. As it has been suggested that the breakup of the coordination cage surrounding a given molecule occurs as the result of molecular reorientation ${ }^{21}$ the lifetime of these cages should be enhanced considerably at low temperatures.

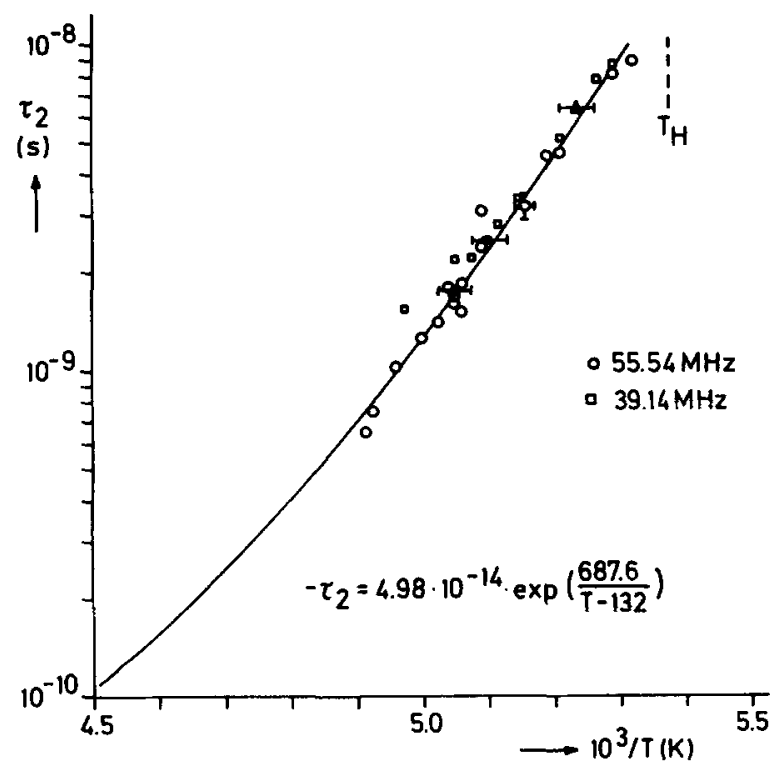

FIG. 3. Temperature dependence of the orientational correlation times $\tau_{2}$ as calculated from the ratio of Eqs. (6) and (7) using the experimental ratio $T_{1} /$ $T_{2}$ obtained at $55.54 \mathrm{MHz}(\mathrm{O})$ and $39.14 \mathrm{MHz}(\square)$. Filled symbols give $\tau_{2}$ calculated from the minimum condition $\omega_{0} \cdot \tau_{2}=0.6158$ with $\omega_{0}$ $2 \pi=55.54 \mathrm{MHz}(0), \omega_{0} / 2 \pi=39.14 \mathrm{MHz}(\square)$, and $\omega_{0} / 2 \pi=15.35 \mathrm{MHz}$ (A). The solid line represents the $T$ dependence of $\tau_{2}$ according to Eq. (14) with parameters as given in Fig. 2. $T_{H}$-homogeneous nucleation temperature at $p=225 \mathrm{MPa}$.
${ }^{c}$ Calculated from Eq. (6) with $\omega_{0} \cdot \tau_{2}=0.6158$

${ }^{\mathrm{d}}$ See Ref. 3.

\section{The deuterium quadrupole coupling constant}

After having calculated the correlation times from $T_{1} /$ $T_{2}$, one can insert these data into either Eqs. (6) or (7) to get an estimate of $\mathrm{DQC}_{\text {eff }}$ in the dispersion region. The resulting values are listed in Table I. They are consistently lower than the values in ice II $(220-225 \mathrm{kHz}),{ }^{22}$ the stable ice polymorph under the conditions of our experiments. Also there is an apparent tendency for $\mathrm{DQC}_{\text {eff }}$ to shift to lower values with increasing temperature, starting very near to $T_{H}$ with a value similar to that found in ice II. Whether this apparent $T$ dependence of $\mathrm{DQC}_{\text {eff }}$ is real or simply an artifact cannot be decided at present because of the fairly large error $(\triangle \mathrm{DQC} /$ $\mathrm{DQC} \simeq 0.2$.

Additional values of $\mathrm{DQC}_{\text {eff }}$ can be obtained from $T_{1}$ at $T_{\min }\left(\omega_{0}\right)$ by inserting $\omega_{0} \tau_{2}=0.6158$ in Eq. (6) and are given in Table II. Their precision is much higher as only the uncertainty of $T_{1}$ contributes $(\triangle \mathrm{DQC} / \mathrm{DQC} \simeq 0.02)$. But they also show a large scatter. Both sets of $\mathrm{DQC}_{\mathrm{efrs}}$ indicate a small systematic error in the $T_{1}$ data measured at $39.14 \mathrm{MHz}$ around $T_{\min }\left(\omega_{0}\right)$, although these have been measured several times with two different samples. At present any observed $T$ dependence of $\mathrm{DQC}_{\text {eff }}$ is within the limits of error.

The fairly low value of $\mathrm{DQC}_{\text {eff }}(\sim 200 \mathrm{kHz})$ which is consistently lower than the corresponding solid state DQC remains puzzling and one has to investigate what mechanisms could influence the effective $D Q C$ in liquid water. During the formation of a $\mathrm{H}$ bond the $\mathrm{O}-\mathrm{D}$ bond lengthens according to a well-established correlation between the $O-D$ and $\mathrm{O}-\mathrm{O}$ distances. ${ }^{23}$ Because of the $r^{-3}$ dependence of the field gradient on distance, the lengthening of the O-D bond has a greater effect in lowering the DQC in asymmetric $\mathrm{H}$ bonds than the more distant charge at the oxygen nucleus of the neighboring molecule. ${ }^{12,13}$ The size of the efg is thus correlated with geometrical parameters of the $\mathrm{H}$ bond. ${ }^{19,24,25}$ Almost linear relations between the DQC and $R_{\mathrm{O}_{-\mathrm{D}}}^{-3}, R_{\mathrm{O}}^{-3} \mathrm{O}$, and $R_{D \cdots}^{-3}$, respectively, have been found. ${ }^{23}$ Thus DQC decreases with increasing bond length $R_{\mathrm{OD}},{ }^{24}$ with decreasing next neighbor distance $R_{\mathrm{O}-\mathrm{O}},{ }^{24}$ and with decreasing $R_{\mathrm{D}_{1} \mathrm{O}} \cdot{ }^{24}$ The dependence of DQC on the $\mathrm{H}$ bond angle $\mathrm{O}-\mathrm{D} \ldots \mathrm{O}$ seems to be only weak. ${ }^{12,23,26}$ Also there is a strong linear correlation between DQC and the stretching frequency $v_{\mathrm{OD}} \cdot{ }^{24,26}$ Though these correlations can be established with solid state data only, they encompass such a large range of H-bond configurations that one can expect them to include all bonding situations which will possibly occur in liquid water. Furthermore, these $\mathrm{H}$-bond parameters are intercorrelated as is shown in the case of $R_{\mathrm{O}-\mathrm{D}}$ vs $R_{\mathrm{O}} \ldots{ }_{\mathrm{O}}{ }^{23}$ and O$\widehat{\mathrm{D}}$... O vs $R_{\mathrm{O}} \ldots \mathrm{O} \cdot{ }^{25}$ Also one should note that the experimen- 
tally determined DQC used to establish these correlations are librationally averaged quantities. In addition the geometrical parameters and their correlated physical quantities such as the DQC are seldom measured at the same temperature. Thus these correlations can only establish qualitative trends. However, their essential correctness has been confirmed by theoretical calculations, ${ }^{12,13,23}$ which exhibited the same pattern of correlation curves.

In addition to these static interdependencies there are dynamic effects influencing $D Q C_{\text {eff }}$. In molecular crystals and crystal hydrates a reduction of DQC with increasing temperature is observed frequently. ${ }^{19}$ An explanation has been given by Bayer ${ }^{27}$ and by Woessner and Gutowsky ${ }^{28}$ in terms of a librational averaging of the DQC. This effect is explicitly taken into account in the derivation of Eqs. (6) and (7). It depends on the amplitude of oscillation, which in turn is influenced by the degree of distortion of an $\mathrm{H}$ bond.

As the various geometric parameters and the amplitudes of oscillation are determined by forces due to interactions with neighboring molecules, it appears reasonable to assume that the correlation of DQC with these parameters will be the same whether the changes are caused by varying the temperature and the pressure or by changing the phase. Then one may use these correlations to perform an order of magnitude estimate of the variation of the DQC that could possibly be induced within the range of temperatures and pressures relevant to the present experiments. Hydrostatic pressure, to begin with, causes a small contraction of $R_{0 \ldots o}$ with a slope of ${ }^{29,30}-0.01 \AA / \mathrm{kbar} \leqslant\left(\partial R_{\mathrm{O} \ldots \mathrm{O}} / \partial p\right)_{T}$ $\leqslant-0.003 \AA / \mathrm{kbar}$ leading to an increase in $R_{\mathrm{O}-\mathrm{D}}$ according to a well-established correlation of $R_{\mathrm{O}-\mathrm{D}}$ with $R_{\mathrm{O} \ldots \mathrm{O}} \cdot{ }^{23} \mathrm{~A}$ rough estimate of the pressure effect on DQC can be obtained if we use the correlation of DQC with the stretching frequency $\tilde{v}_{\mathrm{OD}}{ }^{24}$ :

$$
\operatorname{DQC}(\mathrm{kHz})=0.173 \tilde{v}_{\mathrm{OD}}\left(\mathrm{cm}^{-1}\right)-205
$$

and $\partial \tilde{v}_{\mathrm{OD}} / \partial R_{\mathrm{O}} \ldots \mathrm{O}=770 \mathrm{~cm}^{-1} / \AA$ as given by Whalley. ${ }^{29}$ Using $\partial R_{\mathrm{O}} \ldots \mathrm{O} / \partial p=-0.01 \AA / \mathrm{kbar}^{30}$ gives $\partial \mathrm{DQC} / \partial p=$ $\sim 1.33 \mathrm{kHz} / \mathrm{kbar}$, thus yielding $\Delta \mathrm{DQC} \simeq-3 \mathrm{kHz}$ for a pressure increase of $\Delta p=225 \mathrm{MPa}$.

As the temperature is raised translational and hindered rotational motions will increase in amplitude, and, since $\mathrm{H}$ bond interactions have both distance and angular dependence, the effect will be to reduce, on the average, the strength and ordering effects of these interactions. Concomitantly $\boldsymbol{R}_{\mathrm{O} \ldots \mathrm{O}}$ increases, causing $\boldsymbol{R}_{\mathrm{O}-\mathrm{D}}$ to decrease and thus DQC to rise. Again a rough estimate can be obtained by the correlation of DQC with $\tilde{v}_{\mathrm{OD}}$ and the variation of the hydrogen bonded component frequency with temperature as given by Walrafen ${ }^{31}: \quad \partial \tilde{v}_{\mathrm{OD}} / \partial T \simeq 0.2646 \mathrm{~cm}^{-1} / \mathrm{K}$. Using $\tilde{v}_{\mathrm{OD}}(289 \mathrm{~K})=2516 \mathrm{~cm}^{-1}$ one obtains $\Delta \mathrm{DQC} / \Delta T=0.046$ $\mathrm{kHz} / \mathrm{K}$, yielding $\Delta \mathrm{DQC}(289 \mathrm{~K} \rightarrow 198 \mathrm{~K}) \cong-4 \mathrm{kHz}$.

Whereas the static correlations predict a modest increase of DQC with raising the temperature, the librations cause a reduction of DQC at higher temperatures. Once again we are interested in an order of magnitude estimate of this effect. To lowest order in the mean square angular deviation $\left\langle\theta^{2}\right\rangle$ from the equilibrium $O-D$ direction the averaged DQC is obtained from Eqs. (10) and (11) with $\eta=0$ as

$$
\mathrm{DQC}_{\mathrm{eff}}=\left(\frac{e^{2} q Q}{h}\right) \cdot\left(1-3\left\langle\theta_{l F}^{2}\right\rangle\right)^{1 / 2}
$$

with $\left(e^{2} q Q / h\right)$ the instantaneous value in the absence of librations. Treating the latter as harmonic oscillations gives

$$
\begin{aligned}
\left\langle\theta^{2}\right\rangle & =\frac{1}{2} \frac{\hbar}{I \omega_{1}} \operatorname{coth}\left(\frac{\hbar \omega_{1}}{2 k T}\right) \\
& \simeq \frac{1}{2} \frac{\hbar}{I \omega_{1}}, \quad \text { for } T \lesssim 200 \mathrm{~K} \\
& \simeq 0.02 \text { if } \omega_{1} \simeq 10^{14} \mathrm{~s}^{-1}, \\
I=3 & \left(\sum_{i=1}^{3} \frac{1}{I_{i}}\right)=3.06 \times 10^{-47} \mathrm{~kg} \mathrm{~m}^{2}
\end{aligned}
$$

corresponding to a rms amplitude $\left(\left\langle\theta^{2}\right\rangle\right)^{1 / 2} \simeq 8 \mathrm{deg}$.

Because one does not know the size of $\left(e^{2} q Q / h\right)$ in supercooled water near $T_{H}$, one cannot calculate $\mathrm{DQC}$ eff . A rough estimate of this quantity may be obtained from the known value of $\mathrm{DQC}_{\text {eff }}$ in ice II, ${ }^{22}$ the stable ice modification under the conditions of pressure and temperature given. With an average $\mathrm{DQC}_{\text {eff }}(77 \mathrm{~K}) \simeq 223 \mathrm{kHz}$ one obtains a static value of $230 \mathrm{kHz}$, again using $\left\langle\theta^{2}\right\rangle \simeq 0.02 \mathrm{rad}^{2}$. In order for $\mathrm{DQC}_{\text {eff }}$ to be reduced to $\sim 200 \mathrm{kHz}$ near $T_{H}$ in the liquid one would need $\left\langle\theta^{2}\right\rangle \simeq 0.081 \mathrm{rad}^{2}$ equivalent to a rms angular deviation of $\sim 16 \mathrm{deg}$ which is $\sim 100 \%$ larger than in the solid state and may indicate a larger anharmonicity of the oscillations in the liquid state. Thus an effective DQC of $\sim 200 \mathrm{kHz}$ in supercooled liquid water, as deduced from the experiments, appears reasonable.

As the librational averaging mechanism predicts a $T$ dependence just opposite to what follows from correlations with geometric H-bond parameters of the quasistatic random network it seems safe to suggest that the $\mathrm{DQC}_{\text {eff }}$ will exhibit a modest $T$ dependence in liquid water at low temperatures. Therefore the $\mathrm{DQC}_{\text {eff }}$ can be considered approximately as a $T$ - and $p$-independent parameter.

\section{The temperature dependence of the relaxation times}

The $T$ dependence of the relaxation rates is therefore completely determined by the correlation times $\tau_{2}$. The empirical equation known as the VTF or Doolittle equation $^{32-34}$ is well known for its ability to describe the temperature dependence of transport properties in such diverse systems as molecular liquids, polymers, ionic salts and solutions, and metallic glasses. It has been shown earlier, ${ }^{3}$ that the VTF equation

$$
\tau_{2}(T)=\tau_{20} \exp \left[B /\left(T-T_{0}\right)\right]
$$

applies to liquid water under high hydrostatic pressure also. At the glass transition, characterized by the ideal glass transition temperature $T_{0}$ the liquid becomes arrested in an amorphous structure with the molecules being unable to explore phase space via diffusion. ${ }^{35-43}$ The experimentally determined glass transition temperature $T_{g}$ is, for kinetic reasons, found always $10-20 \mathrm{~K}$ above $T_{0}{ }^{44,45}$ Measurements of $T_{g}$ in aqueous binary mixtures led to an extrapolated glass transition temperature $T_{g}(0.1 \mathrm{MPa})=144 \mathrm{~K}$ in heavy water, ${ }^{46}$ and to $T_{g}(0.1 \mathrm{MPa})=140 \mathrm{~K}^{47}$ and $T_{g}(200$ $\mathrm{MPa})=146 \mathrm{~K}$ in $\mathrm{H}_{2} \mathrm{O}^{48,49}$ Also an ideal glass transition temperature $T_{0}(0.1 \mathrm{MPa})=130 \mathrm{~K}$ has been evaluated by 
Kanno and Angell ${ }^{48,49}$ from the $T$ dependence of the specific heat at constant pressure $C_{p}$.

After insertion of Eq. (20) into Eq. (12) an unconstrained nonlinear least squares fit (N.A.G. E 04 FCF) was performed with the spin-lattice relaxation rates obtained at all three fields and with $\mathrm{DQC} C_{\text {eff }}, \tau_{20}, B$, and $T_{0}$ as adjustable parameters. This fit gave $\mathrm{DQC}_{\text {eff }}=201.2 \mathrm{kHz}$, $\tau_{20}=4.98 \times 10^{-14}(\mathrm{~s}), B=687.6(\mathrm{~K})$, and $T_{0}=132(\mathrm{~K})$. The effective DQC has the value expected from our discussion and $T_{0}$ agrees with the range of values reported in the literature. ${ }^{6}$ The preexponential factor $\tau_{20}$ in the VTF equation has been found to correspond to an average period of liberation of the molecules. ${ }^{3,4}$ Hence $\tau_{20}$ characterizes fast orientational fluctuations of water molecules about their equilibrium orientation in the quasistatic random H-bond network, whilst the orientational correlation time $\tau_{2}$ describes the slower tumbling around of the molecule.

With the parameters from the fit and Eq. (7) the $T$ dependence of the spin-spin relaxation times is correctly predicted as can be seen in Fig. 2. This fact again underscores the essential correctness of Eqs. (6) and (7) deduced with an orientational correlation function which is exponential at long times. Thus the description of single-molecule reorientation in liquid water as an isotropic process is seen to work remarkably well even at temperatures close to $T_{H}$. For water at higher temperatures the same conclusion has previously been drawn from the pressure and temperature dependencies of deuterium and oxygen-17 nuclear magnetic relaxation. ${ }^{50}$ Undoubtedly this isotropy of the water molecule reorientation is due to the locally tetrahedral symmetry of the intermolecular potential in liquid water. However, at sufficiently low temperatures the fitted $T_{1}$ curves are seen to deviate slightly, but systematically, from the experimental data (Fig. 2). The experimentally found $T_{1}$ vary less dramatically with temperature than predicted by Eq. (6) and a constant value of $\mathrm{DQC}_{\mathrm{eff}}$. In principle, this small deviation could be due to a temperature dependence in $\mathrm{DQC}_{\text {eff }}$ (cf. above), but the more likely explanation is that the water molecule reorientation is not completely isotropic, so that more than one correlation time or even a continuous distribution of correlation times has to be invoked for an accurate description. ${ }^{51}$ It is worth noting, that, with a given coupling constant, a reorientational model involving more than one correlation time predicts a larger value of $T_{1}$ at $T_{\min }$ than does the simple isotropic model. Thus, $\mathrm{DQC}$ eff calculated within the isotropic model should be regarded as a lower limit to its true value.

\section{SUMMARY AND CONCLUSIONS}

Spin-lattice relaxation time $\left(T_{1}\right)$ measurements performed recently ${ }^{3}$ under varying hydrostatic pressure in supercooled heavy water at a frequency of $15.35 \mathrm{MHz}$ exhibited a minimum in the $T_{1}(T)$ curve at a pressure $p=225 \mathrm{MPa}$ and a temperature $\sim 5 \mathrm{~K}$ above the homogeneous nucleation temperature $T_{H}$. These results indicated that spin-lattice and spin-spin relaxation rates would become frequency dependent in supercooled liquid water under high hydrostatic pressure at sufficiently low temperatures. The experiments reported here have been undertaken to substantiate this sug- gestion and to gain further insight into the dynamics of orientational fluctuations in liquid water by sampling the spectral density function $g(\omega)$ at different frequencies and various temperatures. Starting with a decomposition of orientational motions of water molecules into fast librational oscillations and slower diffusive motions it was assumed that these motions are statistically independent because they occur on largely different time scales. Describing the slow diffusive reorientations via a time correlation function which is exponential at long times with a characteristic time constant, the orientational correlation time $\tau_{2}$, simple expressions for the relaxation rates were deduced, including the effect of both the fast and the slow motions of the molecules. The fast oscillations are seen mainly to reduce the instantaneous value of the deuterium quadrupole coupling constant which a deuteron possesses in the absence of oscillations in a water molecule engaged in $\mathbf{H}$-bond interactions within the quasistatic random network. Any additional contribution from the fast oscillations to the spectral density function $g\left(\omega_{0}\right)$ was found to be negligible. This conclusion is corroborated by the numerical agreement of the experimental ratio of $T_{1} / T_{2}$ at $T_{\min }\left(\omega_{0}\right)$ with the prediction of Eqs. (6) and (7). Also a least squares fit of the $T_{1}$ data to Eq. (6) yielded parameters which, after insertion into Eq. (7), correctly predicted the $T$ dependence of the spin-spin relaxation times $\left(T_{2}\right)$ as deduced from linewidth measurements.

The size of $\mathrm{DQC}_{\text {eff }}$ has been found to be smaller than the corresponding solid state value. It is argued that the reduction from the solid state value may be explained by the larger amplitude of the librations in the liquid and their effect upon the component of the efg tensor along the equilibrium direction of the OD bond in the quasistatic random $\mathrm{H}$ bond network.

The $T$ dependence of the relaxation rates is dominated by the variation of the correlation time $\tau_{2}$ and this dependence is well described by the VTF equation ${ }^{32}$ in accord with results obtained earlier. ${ }^{50}$ The glass transition temperature $T_{0}$ is in good agreement with estimates available in the literature. The preexponential factor $\tau_{20}$ in the VTF equation is of the same magnitude as the period of libration and characterizes the fast orientational fluctuations of the water molecules in the potential wells of the quasistatic random network. As the temperature is lowered the time scale for structural rearrangements of the $\mathrm{H}$-bond topology becomes progressively longer and the molecules become fixed in given orientations for increasingly longer time spans, executing small amplitude oscillations about their mean orientations.

\section{ACKNOWLEDGMENTS}

It is a pleasure to thank Dr. B. Halle and Dr. H. Wennerström for stimulating discussions and comments to our manuscript. We are also indebted to $H$. Lilja and L. Nilsson for modifying the spectrometers, to R. Knott and S. Heyn for their expert technical assistance, and to Dr. T. Drakenberg for sharing his vast NMR know-how with us. E. W. Lang likes to thank Professor B. Lindman and Professor S. Forsen for their kind hospitality during his stay at the Physical Chemistry Department of the University of Lund. The least squares fitting of the data was done at the computer 
center of the University of Regensburg. Financial support from the Swedish Natural Sciences Research Council and the Deutsche Forschungsgemeinschaft is gratefully acknowledged.

${ }^{\prime} \mathrm{C}$. A. Angell, in Water-A Comprehensive Treatise, edited by F. Franks (Plenum, New York, 1982), Vol. 7, p. 1 ff.

${ }^{2}$ E. W. Lang and H.-D. Lüdemann, J. Chem. Phys. 67, 718 (1977).

${ }^{3}$ E. W. Lang and H.-D. Lüdemann, Ber. Bunsenges. Phys. Chem. 84, 462 (1980).

${ }^{4}$ E. W. Lang and H.-D. Lüdemann, Ber. Bunsenges, Phys. Chem. 85, 603 (1981).

${ }^{5}$ E. W. Lang and H.-D. Lüdemann, Ber. Bunsenges. Phys. Chem. 85, 1016 (1981).

${ }^{6} \mathrm{E}$. W. Lang, Dissertation, Universität Regensburg, 1980. Recently $T_{1}\left(T, p, \omega_{0}\right)$ also have been measured in $\mathrm{H}_{2}{ }^{17} \mathrm{O}(26 \%$ and $50 \%$ enriched $)$ and $\mathrm{H}_{2}{ }^{16} \mathrm{O}$ at 360 and $255 \mathrm{MHz}$ by the present authors. These results will be the subject of a subsequent publication.

${ }^{7}$ M. L. Martin, J.-J. Delpuech, and G. J. Martin, Practical NMR Spectroscopy (Heyden, London, 1980).

${ }^{8} \mathrm{D}$. H. Rassmussen and A. P. McKenzie, in Water Structure and the Water-Polymer Interface, edited by H. H. Jellinek (Plenum, New York, 1972), p. 126.

${ }^{9}$ E. W. Lang, R. Rauchschwalbe, and H.-D. Lüdemann, High Temp. High Pressure 9, 519 (1977)

${ }^{10} \mathrm{~A}$. Abragam, The Principles of Nuclear Magnetism (Oxford University, London, 1961), p. $313 \mathrm{ff}$.

"E. A. C. Lucken, Nuclear Quadrupole Coupling Constants (Academic, London, 1969).

${ }^{12}$ L. G. Butler and Th. L. Brown, J. Am. Chem. Soc. 103, 6541 (1981)

${ }^{13}$ L. Mayas, M. Plato, C. J. Winscom, and K. Möbius, Mol. Phys. 36, 753 (1978).

${ }^{14}$ M. Weismann, J. Chem. Phys. 44, 422 (1966)

${ }^{15} \mathrm{H}$. W. Spiess, in NMR-Basic Principles and Progress, edited by P. Diehl, E. Fluck, and R. Kosfeld (Springer, Berlin, 1978), Vol. 15.

${ }^{16} \mathrm{M}$. E. Rose, Elementary Theory of Angular Momentum (Wiley, New York, 1957).

${ }^{17}$ M. Sceats and S. A. Rice, in Water-A Comprehensive Treatise, edited by F. Franks (Plenum, New York, 1982), Vol. 7, p. 83 ff.

${ }^{18}$ B. Halle and H. Wennerström, J. Chem. Phys. 75, 1928 (1981)

${ }^{19} \mathrm{~A}$. Weiss and N. Weiden, in Advances in Nuclear Quadrupole Resonance, edited by J. A. S. Smith (Heyden, London, 1980), Vol. 4, p. 149 ff.

${ }^{20} \mathrm{~J}$.-P. Hansen, in Microscopic Structure and Dynamics of Liquids, edited by J. Dupuy and A. J. Dianoux (Plenum, New York, 1978).

${ }^{21}$ R. W. Impey, P. A. Madden, and I. R. McDonald, Mol. Phys. 46, 513 (1982).
${ }^{22}$ D. T. Edmonds, S. D. Goren, A. L. Mackay, and A. A. L. White, J. Magn. Reson. 23, 505 (1976).

${ }^{23}$ S. D. Goren, J. Chem. Phys. 60, 1892 (1974).

${ }^{24}$ B. Berglund, J. Lindgren, and J. Tegenfeldt, J. Mol. Struct. 43, 179 (1978)

${ }^{25}$ R. Chidambaram and S. K. Sikka, Chem. Phys. Lett. 2, 162 (1968).

${ }^{26}$ D. T. Edmonds, S. D. Goren, and A. A. L. White, J. Magn. Reson. 27, 35 (1977).

${ }^{27}$ H. Bayer, Z. Phys. 130, 227 (1951).

${ }^{28}$ D. E. Woessner and H. S. Gutowsky, J. Chem. Phys. 39, 440 (1963).

${ }^{29}$ E. Whalley, J. Chem. Phys. 63, 5205 (1975).

${ }^{30}$ G. A. Gaballa and G. W. Neilson, Mol. Phys. 50, 97 (1983).

${ }^{31} \mathrm{G}$. E. Walrafen, in Water $-A$ Comprehensive Treatise, edited by F. Franks (Plenum, New York, 1972), Vol. 1, p. 151 ff.

${ }^{32}$ (a) H. Vogel, Z. Phys. 22, 645 (1921); (b) G. Tammann and G. Hesse, Z. Anorg. Allg. Chem. 156, 245 (1926); (c) G. S. Fulcher, J. Am. Chem. Soc. 8, 339 (1925); (d) A. K. Doolittle, J. Appl. Phys. 22, 1471 (1951).

${ }^{33}$ M. Cyrot, Phys. Lett. A 83, 275 (1981).

${ }^{34}$ D. Frenkel and J. P. McTague, Annu. Rev. Phys. Chem. 31, 491 (1980).

${ }^{35}$ M. Goldstein, J. Chem. Phys. 51, 3728 (1969)

${ }^{36}$ M. H. Cohen and G. S. Grest, Phys. Rev. B 20, 1077 (1979).

${ }^{37}$ A. R. Eastwood, J. Chem. Soc. Faraday Trans. 2 77, 1411 (1981).

${ }^{38}$ M. H. Cohen and D. Turnbull, J. Chem. Phys. 31, 1164 (1959).

${ }^{39}$ P. B. Macedo and T. A. Litovitz, J. Chem. Phys. 42, 245 (1965).

${ }^{40}$ G. Adam and J. H. Gibbs, J. Chem. Phys. 43, 139 (1965).

${ }^{41}$ C. A. Angell and D. L. Smith, J. Phys. Chem. 86, 3845 (1982).

${ }^{42}$ C. A. Angell, J. H. R. Clarke, and L. V. Woodcock, Adv. Chem. Phys. 48 , 397 (1981)

${ }^{43}$ J. N. Cape and L. V. Woodcock, J. Chem. Phys. 72, 976 (1980).

${ }^{44}$ C. A. Angell, J. Chem. Educ. 47, 583 (1970).

${ }^{45}$ C. A. Angell and K. J. Rao, J. Chem. Phys. 57, 470 (1972).

${ }^{46}$ H. Kanno, J. Shirotani, and S. Minomura, Bull. Chem. Soc. Jpn. 53, 2079 (1980).

${ }^{47}$ C. A. Angell and E. J. Sare, J. Phys. Chem. 71, 2759 (1967).

${ }^{48} \mathrm{H}$. Kanno and C. A. Angell, J. Chem. Phys. 73, $1940(1980)$.

${ }^{49} \mathrm{H}$. Kanno and C. A. Angell, J. Phys. Chem. 81, 2639 (1977).

${ }^{50} \mathrm{E}$. W. Lang and H.-D. Lüdemann, Angew. Chem. Int. Ed. Engl. 21, 315 (1982).

${ }^{51}$ C. W. R. Mulder, J. Schriever, and J. C. Leyte, J. Phys. Chem. 87, 2336 (1983).

52J. M. Emsley, S. K. Khoo, and G. R. Luckhurst, Mol. Phys. 37, 959 (1979).

${ }^{53}$ B. Ban, J. Magn. Reson. 45, 118 (1981).

${ }^{54}$ To obtain this result the assumption of an isotropic slow motion is unneccessary. A more general derivation can be given within the two-step model of Ref. 18. P. Cummins, G. B. Bacskay, N. S. Hush, E. Engström, and $B$. Halle (to be published).

${ }^{55}$ B. Halle, H. Wennerström, and L. Piculell, J. Phys. Chem. 88, 2482 (1984). 